\title{
Estudo epidemiológico sobre o vírus da imunodeficiência humana (HIV) em indígenas do estado de Roraima entre 2010 a 2018
}

\author{
Epidemiological study about human immunodeficiency virus (HIV) in indigenous at \\ Roraima state among 2010 to 2018
}
Estudio epidemiológico sobre el virus de la inmunodeficiencia humana (VIH) en indígenas del estado Roraima entre 2010 a 2018
Vanessa Fernandes Ribeiro ${ }^{1}$, Ana Paula Barbosa Alves ${ }^{1 *}$, Luiza Brum Argenta ${ }^{1}$, Hosana Carolina dos Santos Barreto'.

\section{RESUMO}

Objetivo: Verificar a prevalência de HIV/AIDS entre indígenas do Estado de Roraima no período de 2010 a 2018. Métodos: Trata-se de um estudo descritivo, epidemiológico, transversal de método quantitativo. Foram coletados dados secundários, proveniente do Sistema de Informação do Ministério da Saúde (MS), do DATASUS, com a evolução temporal das taxas de prevalência de AIDS em indígenas do Estado de Roraima, restritas ao período de 2010 a 2018. Foram analisadas as varáveis sócio demográficas: distribuição por município de residência, faixa etária, escolaridade e categoria de exposição. Os dados foram organizados e tabulados por meio de planilhas do Microsoft Excel 2010, juntamente com a elaboração de gráficos. Resultados: Constatou-se que os maiores percentuais de infecção HIV/AIDS entre indígenas estão entre o sexo masculino, e as faixas etárias mais acometidas são de 20 a 39 anos, com escolaridade entre $1^{\underline{a}}$ a $4^{a}$ serie incompleta com $25 \%$ de casos, e ensino médio com $16 \%$ de casos, respectivamente. A principal categoria de exposição é a heterossexual. Conclusão: A prevalência da AIDS é uma realidade entre os povos indígenas e que devido a sua incidência e pelo seu status de cronicidade e por não haver cura o número de casos está aumentando constantemente.

Palavras-chave: Epidemiologia, Indígenas, HIV.

\begin{abstract}
Objective: Verify the prevalence of HIV/AIDS among indigenous in Roraima State in the period of 2010 to 2018. Methods: treats of one describing, epidemiological, transversal of a quantitative method. Were colected the secondary data from Information System of Ministry of Health (MH), from DATASUS, with the elapse of time the AIDS prevalence rates in indigenous has increased in Roraima State, restricted to the period of 2010 to 2018. Were analyzed the social demographics variables: distribution by municipality of residence, age group, schooling and exposure category. The data has been organized and tabulated by spreadsheets in Microsoft Excel 2010, along with graphing. Results: Has been noticed that the higher percentages of infections by HIV/AIDS in indigenous are of male indigeneous, and the age group most affected are of 20 to 39 years, with the schooling in $25 \%$ of the cases with an incomplete elementary school and $16 \%$ with higher school. The main category of exposure is the heterosexual. Conclusion: The prevalence of AIDS is present among the indigenous peoples, due to it's incidence and chronicity, with no cure the number of cases increases constantly.
\end{abstract}

Keywords: Epidemiological, Indigenous, HIV.

${ }^{1}$ Universidade Federal de Roraima. Boa Vista - RR. *E-mail: paula.alves@ufrr.br 


\section{RESUMEN}

Objetivo: Verificar la prevalencia del VIH/SIDA entre indígenas del estado de Roraima en el periodo de 2010 a 2018. Métodos: Se trata de un estudio descriptivo, epidemiológico, transversal de método cuantitativo. Fueron recolectados datos secundarios del Sistema de Información del Ministerio de Salud (MS), de DATASUS, con la evolución temporal de las tasas de prevalencia del SIDA en indígenas del Estado de Roraima, restringidas al período de 2010 a 2018. Fueron analizadas las variables sociodemográficas: distribución por municipio de residencia, faja de edad, escolaridad y categoría de exposición. Los datos fueron organizados y tabulados a través de planillas de Microsoft Excel 2010, junto con la elaboración de gráficos.

Resultados: Se constató que los mayores porcentajes de infección VIH/SIDA entre indígenas está entre el sexo masculino, y las fajas de edad más afectadas son de 20 a 39 años, con escolaridad entre $1^{1}$ y $4^{\text {a }}$ grado incompleta con $25 \%$ de casos, y escuela secundaria con $16 \%$ de casos, respectivamente. La principal categoría de exposición es heterosexual. Conclusión: La prevalencia del SIDA es una realidad entre los pueblos indígenas, que debido a su incidencia y por su estado de cronicidad y por no haber cura, el número de casos aumentando constantemente.

Palabras clave: Epidemiología, Indígena, VIH.

\section{INTRODUÇÃO}

A epidemiologia é a ciência que estuda o processo saúde-doença na sociedade; promoção ou recuperação da saúde individual ou coletiva produzindo informação e conhecimento para tomada de decisão (ALMEIDAFILHO N e MEDRONHO RA, 2009). É um método para o estudo da distribuição de um problema de saúde na população e para investigação das razões desta distribuição (PEREIRA MG, 2008).

Assim, destaca-se o papel importante da vigilância epidemiológica para medir as ações de controle da epidemia do Vírus da Imunodeficiência Humana (HIV) e da Síndrome da Imunodeficiência Adquirida (Aids) e subsidiar o planejamento de estratégias de intervenção (SZWARCWALD CL, et al., 2016).

O HIV é o causador da Aids que é um problema de saúde pública o qual está sendo enfrentado mundialmente devido a sua pertinência pandêmica. No Brasil, em 2018, foram diagnosticados 43.941 novos casos de HIV e 37.161 casos de aids com uma taxa de detecção de 17,8/100.000 habitantes (2018), totalizando, no período de 1980 a junho de 2019, 966.058 casos de aids detectados no país (BRASIL MS, 2019, BRASIL MS, 2018).

Sua descoberta se deu a partir dos anos 1980 nos Estados Unidos da América (EUA), através da identificação de casos de "sarcoma de Kaposi" e de uma pneumonia típica (causada por Pneumocystis carinii), em homens jovens, saudáveis, grande parte homossexuais das cidades como Nova York, São Francisco e Los Angeles (BASTOS FI, 2006).

No Brasil, o primeiro caso foi identificado no Estado de São Paulo, ainda em 1980 (BRASIL MS, 2006). Entre a população indígena, os primeiros registros ocorreram em 1988, e desde então os números de casos tem aumentado a cada ano (BRASIL MS, 2002).

Os fatores que possibilitam a interiorização do vírus entre a população indígena no Brasil podem estar relacionados a exploração de garimpo, extração de madeira em terras indígenas; a construção de barragens, rodovias e projetos agropecuários; turismo; trânsito em regiões de fronteira; populações indígenas assentadas em áreas urbanas. Ressalta-se que a população que tem maior contato com a sociedade civil é a mais acometidas por Infecções Sexualmente Transmissíveis (ISTs) (BRASIL MS, 2000; CARVALHO NS, et al., 2011).

O HIV pode ser transmitido por diversas formas: transfusões sanguíneas, relação sexual (espermas e secreção vaginal), transmissão vertical (da mãe para o filho na gestação, na hora do parto e amamentação), por acidentes com material perfuro cortante durante o manuseio com sangue e secreções e pelo compartilhamento de seringas e agulhas (TÁVORA LGF, et al., 2014). 
Para a Aids, não existe até o momento uma cura ou vacina, o tratamento é embasado com o uso de antirretrovirais, os quais possuem vários efeitos colaterais, mas podem controlar o avanço da doença, complicações e o risco de morte imediata além de elevar a expectativa de vida das pessoas que convivem com o vírus. Embora estes medicamentos sejam de alto custo, são distribuídos gratuitamente no Brasil desde 1996 pelo Ministério da Saúde.

A Aids ainda traz grandes impactos na sociedade atual, principalmente em populações considerados vulneráveis devido às condições sociais, culturais e biológicas (POLEJACK L e SEIDL EMF, 2010). Ressaltase que atualmente com os avanços da medicina, a Aids alcançou um perfil epidemiológico crônico, no entanto a adesão ao tratamento continua sendo um grande desafio, juntamente com a depressão que é causa de impacto na terapêutica desta enfermidade (OLIVEIRA EH, 2019).

Ressalta-se que a preocupação com essa doença é em consequência da notificação de casos no período de 2007 a 2018, totalizando 247.795 casos de infecção pelo HIV no Brasil. Em relação à distribuição proporcional dos casos de Aids declarados de 1980 até junho de 2018, apresenta uma centralização nas regiões Sudeste e Sul, respectivamente de $51,8 \%$ e $20,0 \%$ do total de casos; nas regiões Nordeste, Norte e Centro-Oeste correspondem a $15,8 \%, 6,4 \%$ e $6,1 \%$ do total dos casos, respectivamente.

Observa-se nos últimos cinco anos (2013 a 2017), a região Norte vem apresentando uma média crescente de 4,4 mil casos ao ano; em relação ao Nordeste, 9,0 mil; o Sudeste, 16,1 mil; o Sul, 8,2 mil; e o CentroOeste, 2,9 mil (BRASIL MS, 2018).

Roraima é o estado localizado no extremo norte do Brasil e possui uma população total de 450.479 habitantes divididos entre os 14 municípios: Alto Alegre, Amajarí, Bonfim, Cantá, Caracaraí, Caroebe, Iracema, Mucajaí, Normandia, Pacaraima, Rorainópolis, São João da Baliza, São Luiz e Uiramutã. Além disso, há 49.637 indígenas, que ocupam uma área de 10.370.676 hectares, tendo como os principais povos (etnias): Macuxi, Taurepang, Ingarikó, Yanomami, Y'ekuana, Sanumá, Sapará, Patamona, Wapixana, Waimiri-Atroari e Wai-Wai (IBGE CENSO DEMOGRÁFICO, 2010; CARVALHO F, et al.,2008).

Entre a população indígena não é diferente, a infecção pelo HIV/Aids é um agravo que tem ameaçado um grande número de comunidades indígenas em todo o Brasil (BRASIL MS, 2002). No entanto, evidencia-se que a maior vulnerabilidade desta população à transmissão do HIV/AIDS, está relacionada a más condições de vida, menor nível socioeconômico, educacional, exclusão social e dificuldade de acesso aos serviços de saúde (NEGIN J, et.al., 2015). Diante disso, questiona-se: qual a prevalência do HIV/AIDS entre a população indígenas do Estado de Roraima entre os anos de 2010 a 2018?

Deste modo, esta análise possui uma grande relevância, pois se trata de um estudo epidemiológico que permite conhecer alguns aspectos relacionados à distribuição da infecção do HIV entre a população indígena do Estado de Roraima. Neste sentido, subsidia e traz conhecimentos para aprimorar ações e programas de prevenção e controle do HIV/Aids no Estado.

O objetivo deste estudo é verificar a prevalência de HIV/Aids entre a população indígena do Estado de Roraima, a partir de um estudo de dados secundários do Ministério da Saúde (MS) disponibilizados através do Departamento de Informática do Sistema Único de Saúde (DATASUS) sobre notificações de Aids no período de 2010 a 2018.

\section{MÉTODOS}

Trata-se de um estudo descritivo, epidemiológico, transversal. A pesquisa descritiva busca descrever as características de determinada população (GIL AC, 2008). O trabalho epidemiológico é empírico, quantitativo, baseado na coleta ordenada de informações sobre a saúde em uma população determinada (BLOCH KV e COUTINHO ESF, 2009).

A pesquisa transversal pode ser de incidência e prevalência (KLEIN CH e BLOCH KV, 2009). A incidência pesquisa determinada doença ou agravo em grupos de casos novos. É dinâmica, varia com o tempo e em diferentes espaços. A prevalência analisa casos existentes (antigos e novos) de uma doença ou agravo em 
local e tempo determinado; é estática, e essencialmente transversal, seria um registro, uma foto, em que os fenômenos são observados em mesmo momento histórico (COSTA AJL e KALE PL, 2009). Por se tratar de um estudo com dados secundários não houve a necessidade de submissão ao Comitê de Ética e Pesquisa da UFRR, porém foi solicitada a anuência, no sentindo de disponibilizar as informações e poder usar o nome das instituições caso necessário no trabalho.

Foram coletados dados secundários provenientes do Sistema de Informação do Ministério da Saúde do DATASUS com a evolução temporal das taxas de prevalência de HIV/Aids entre indígenas do estado de Roraima, restritas ao período de 2010 a 2018. Foram analisadas as varáveis sócio demográficas (distribuição por município de residência, faixa etária, escolaridade e categoria de exposição). Os dados foram organizados e tabulados por meio de planilhas do Microsoft Excel 2010, e analisados através de gráficos e tabelas para melhor compreensão.

A prevalência busca saber com que frequência um evento ou agravo está ocorrendo em uma determinada população, e é calculada pelo número de casos existentes (casos novos + antigos) da doença dividido pela população de interesse. Os seus valores possíveis estão entre 0 e 1, e são expressos em percentagens (COSTA AJL e KALE PL, 2009).

\section{RESULTADOS e DISCUSSÃO}

Segundo o Ministério da Saúde brasileiro em 2017, o ranking das Unidades da Federação referentes às taxas de detecção de Aids mostrou que os estados de Roraima e Amapá apresentaram as maiores taxas, com 36,8 e 29,8 casos por 100 mil habitantes, respectivamente (BRASIL MS, 2018). O número de pessoas vivendo com HIV/Aids são elevados em Roraima. Apesar de ser o estado com a menor população do país, 0 quantitativo é bastante significativo (RORAIMA, SECRETARIA DE ESTADO DA SAÚDE DE RORAIMA. 2019).

No período compreendido entre os anos de 2010 a 2018 foram registrados 1.113 casos da doença em Roraima, em sua maioria no município de Boa Vista (938 casos), correspondendo $84 \%$ do total, seguido de Rorainópolis e Pacaraima com 29 e 21 casos respectivamente. Neste cenário, Boa Vista se destaca por apresentar o maior índice de prevalência registrado no período, com taxa de 3,30 por mil habitantes, isso em decorrência do município possuir maior população e concentrar as unidades de alta complexidade que notificam os casos no Estado. A intensidade da doença pode ser observada por município de residência a partir da análise da Figura 1.

Figura1 - A Prevalência de Aids no Estado de Roraima no Período de 2010 a 2018, por mil habitantes.

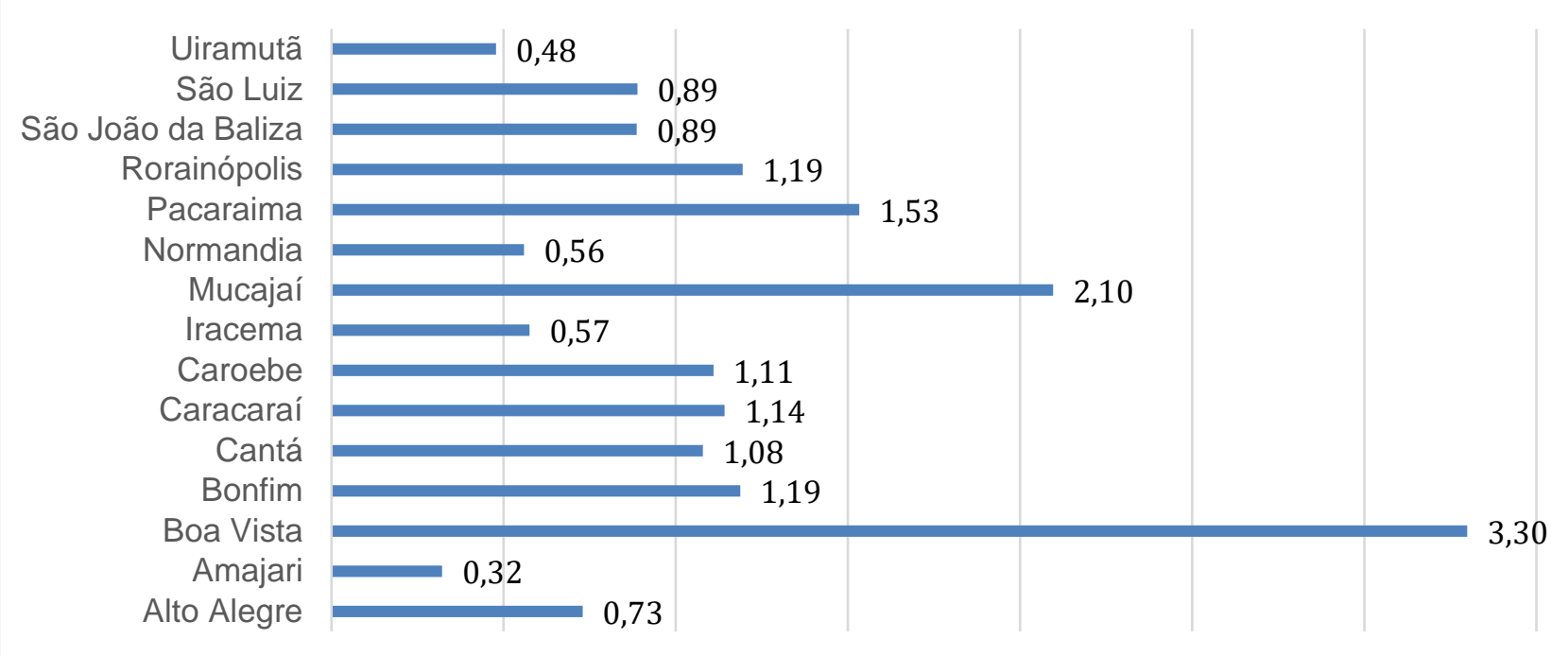

Fonte: MS/DATASUS; Dados consolidados até 30/06/2018. 
Costa AJL e Kale PL (2009) descrevem que a prevalência de uma doença é verificada pela sua incidência e sua duração. Segundo o estudo de Pereira GFM et al. (2019) conforme a prevalência do HIV se consolida na população geral, partes populacionais em condições de maior vulnerabilidade e risco de infecção concentram altas prevalências. Historicamente as populações indígenas são mais vulneráveis epidemiologicamente e socialmente (GARNELO L e PONTES AL, 2012). Neste sentido, Soares JP et al. (2017) afirmam que acrescente prevalência à infeção pelo HIV, se mantem em populações que encaram dificuldades de acesso a informações de prevenção, ao tratamento e a serviços de saúde, vítimas de abuso, violência, privação econômica e social.

Outro fator que pode estar contribuindo para as taxas de prevalência do HIV/aids em Boa Vista em Roraima seria o fenômeno da migração internacional. Amon J e Todrys k (2009) ratificam que os direitos à saúde e prevenção e tratamento ao HIV/Aids dos migrantes deve ser garantido e respeitados, e isso se tornaria uma estratégia para controle e combate dessa pandemia.

Vale ressaltar que os processos migratórios internacionais em todo mundo não são específicos de nossa contemporaneidade. Os sujeitos se movem em busca de novas esperanças e oportunidades econômicas. Outros se deslocam para fugir de conflitos armados, pobreza, insegurança alimentar, perseguição, terrorismo ou violações a Direitos Humanos. Existem, ainda, os que o fazem em resposta as implicações antagônicas de mudanças climáticas, desastres naturais ou demais fatores ambientais (POMPEU GVM e SOUSA RJ, 2019).

Segundo Rodrigues HAN (2015) os fluxos migratórios internos e externos, provindos dos países fronteiriços, contribuem para a disseminação de HIV/Aids em Roraima. Constata-se que desde 2015 houve um incremento expressivo do fluxo de venezuelano para outros países, principalmente para o Brasil onde o estado de Roraima foi a que mais recebeu estes imigrantes. A crise econômica e política deste país resultam como uma das principais causas para a imigração (SIMÕES GF, 2017).

A evolução histórica da doença no estado pode ser observada a partir da Figura 2 na qual os dados chamam atenção para o ano de 2017 onde apresentou uma taxa de 0,52 por mil habitantes, tendo em vista que desde 2013 tem mostrado um crescimento significativo. Houve uma redução em 2018, considerando que estes dados foram coletados até junho de 2018.

Figura 2 - Evolução histórica de Aids no Estado de Roraima no período de 2010 a 2018, por mil habitantes.

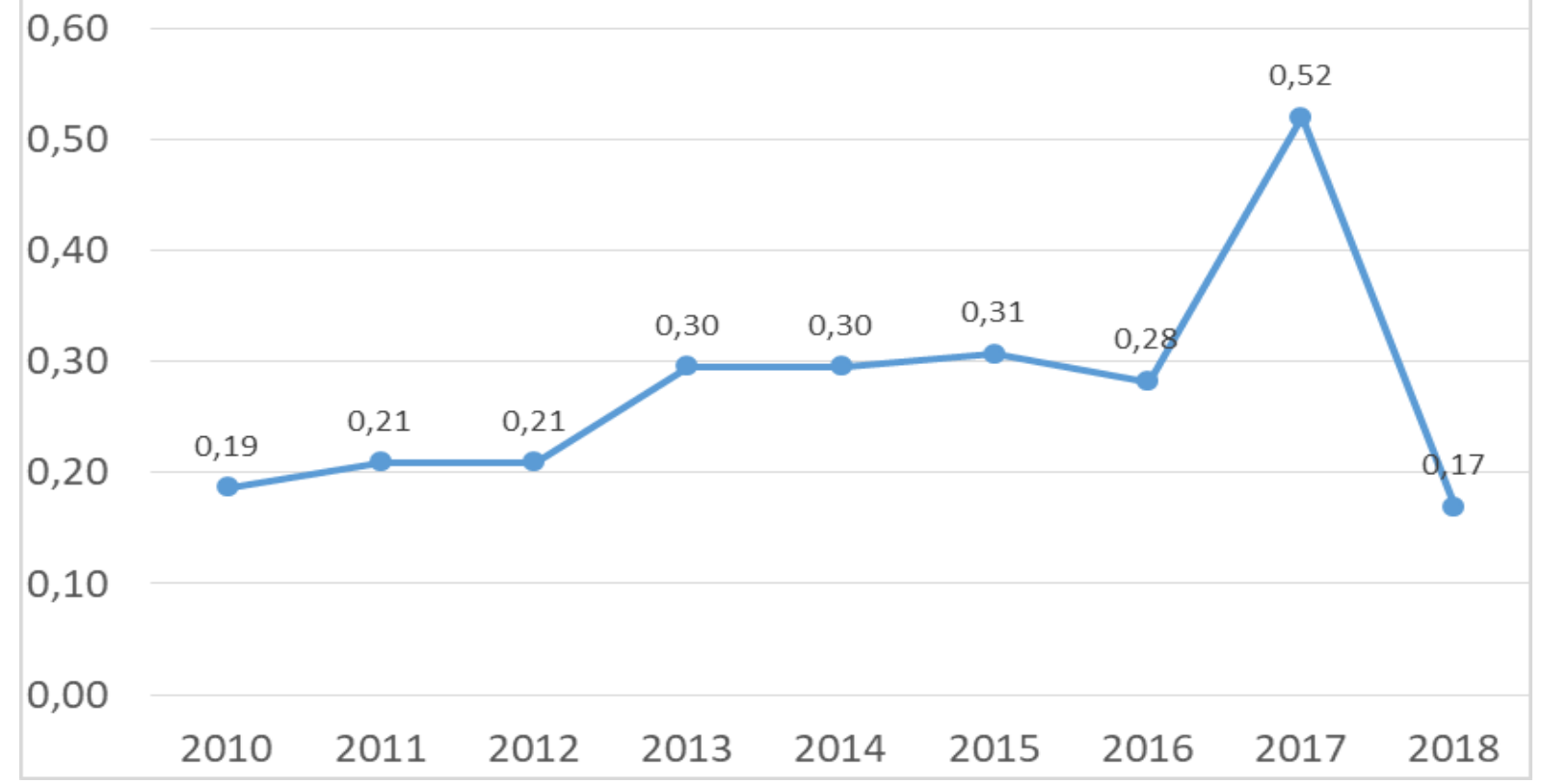

Fonte: MS/DATASUS; Dados consolidados até 30/06/2018. 
É importante ressaltar que, apesar do avanço no número de casos da doença, o programa IST/Aids criado pelo governo federal brasileiro é referência mundial por sua universalização, possibilitando a todos o tratamento gratuito. Porém, a não procura de centros de saúde para a realização do teste anti-HIV tem sido um dos motivos pela qual a doença tem se propagado, já que os mesmos não possuem o conhecimento que são portadores do vírus mesmo em um país que oferece medicamentos para o tratamento. Ainda que estes medicamentos não proporcionem a cura, garantem o controle da doença e prolonga a vida da pessoa portadora do HIV (RODRIGUES HAN, 2015).

Em relação à taxa de prevalência na população indígena do Estado de Roraima no período de 2010 a 2018, a maior taxa de Aids foi observada no município de Pacaraima que representou 0,67 por mil habitantes, seguido de Bonfim com 0,64 e Normandia com 0,34 conforme a Figura 3. Vale ressaltar que o município de Pacaraima faz fronteira com o país da Venezuela e Bonfim com Guiana, um dos fatores que permitem a propagação Aids entre a população indígena do Estado.

Figura 3 - A Prevalência de Aids entre a população Indígena do Estado de Roraima no período de 2010 a 2018, por mil habitantes.

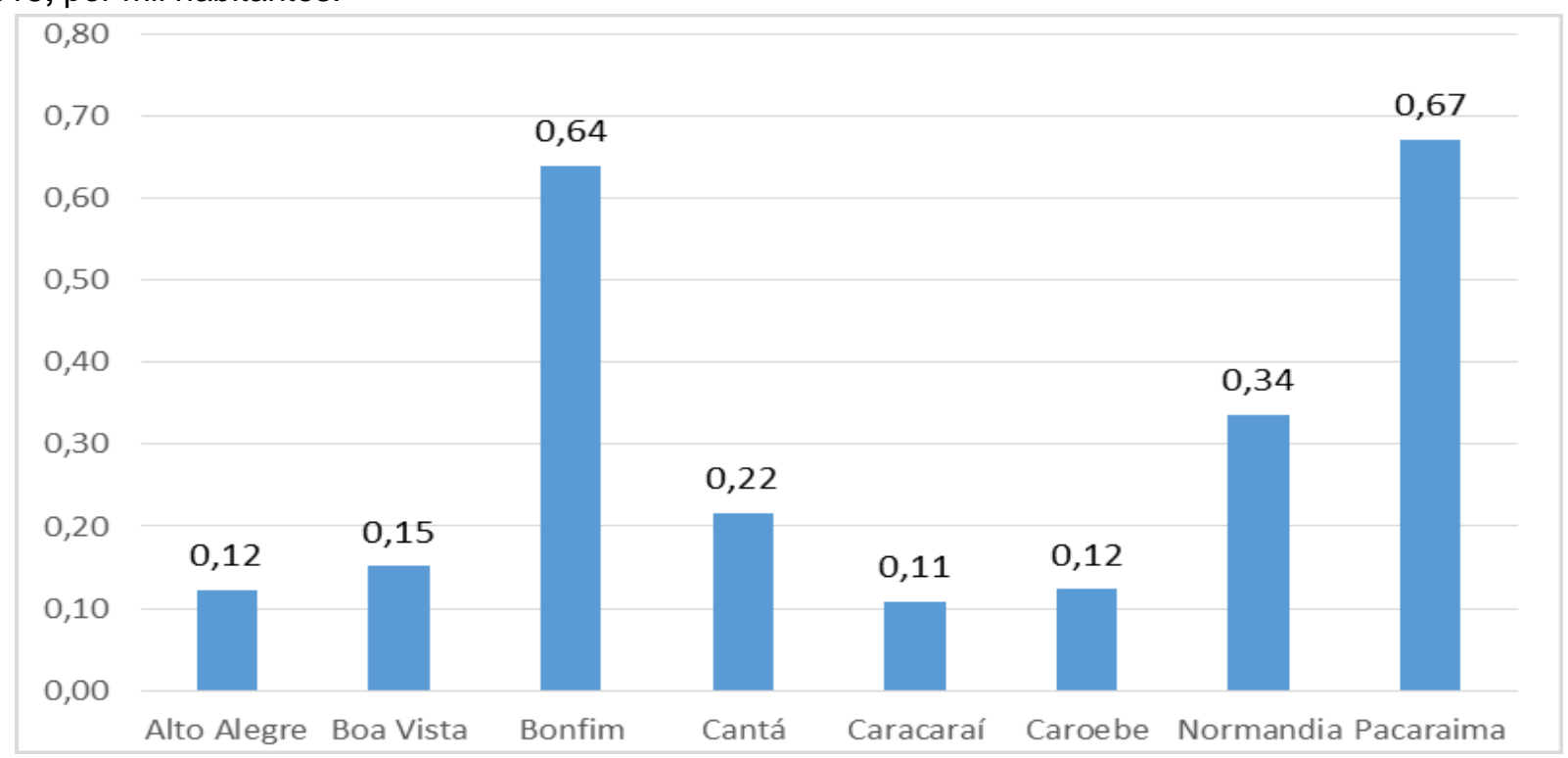

Fonte: MS/DATASUS; Dados consolidados até 30/06/2018.

É importante destacar que dentre os quinze municípios do Estado apenas oito registraram casos de Aids. Este viés leva a refletir se realmente os demais municípios não apresentaram casos de Aids ou não foram registrados no banco de dados. Para Marinho MFS (2008) é necessária uma base de informações confiáveis que sustente e direcione a tomada de decisões na formulação dos planejamentos a fim de tratar dos problemas de saúde.

No que tange as probabilidades de risco entre os povos indígenas, devem ser consideradas e avaliadas várias situações nos contextos epidemiológicos, culturais e sociais de cada povo, bem como: iniciação sexual, frequência e posição social dos homens e mulheres que saem das comunidades, ocorrência de interações entre grupos étnicos distintos, rituais ou eventos que envolvam manuseio de objetos perfuro cortantes sem adequada desinfecção, práticas sexuais de poligamia, poliandria e poligimia, práticas de aleitamento cruzado, alcoolismo, prevalência de ISTS e outros fatores de risco relacionado à infecção pelo HIV (BRASIL MS, 2000). Estudos que descrevam as condições sanitárias das populações indígenas no Brasil ainda são poucos. Este fato é devido à grande dificuldade de levantamento dos dados, devido aos problemas relativos às dificuldades de acesso em consequência às extensas áreas geográficas do país, barreiras culturais, e até mesmo poucos conhecimentos a respeito das comunidades indígenas. Mas se faz um apelo ao estado de vulnerabilidade a vários agravos e doenças que essas populações diferenciadas estão expostas (CARVALHO NS, et al., 2011). 
Em relação à vulnerabilidade, é importante levar em consideração as proximidades das terras indígenas aos centros urbanos, entradas de não indígenas nas comunidades, a inclusão de jovens indígenas nas cidades, o consumo abusivo de bebidas alcoólicas, resistências ao uso do preservativo, poucas informações e acesso aos exames diagnósticos pelos serviços às gestantes e fatores socioeconômicos como pauperização, que caracterizam alguns dos principais fatores de vulnerabilidade as ISTs e HIV/Aids para os povos indígenas (SANTOS VL, et al., 2010).

Na Figura 4, pode ser observada a evolução dos casos notificados de Aids na população indígena do estado de Roraima no período de 2010 a 2018. Constata-se que as maiores taxas foram registradas em 2014 e 2017 representando 0,04 por mil habitantes. No entanto, nota-se que em 2015 houve uma redução para 0,01 da taxa de detecção por mil habitantes.

Figura 4 - Evolução dos casos de AIDS na população Indígena do Estado de Roraima no período de 2010 a 2018, por mil habitantes.

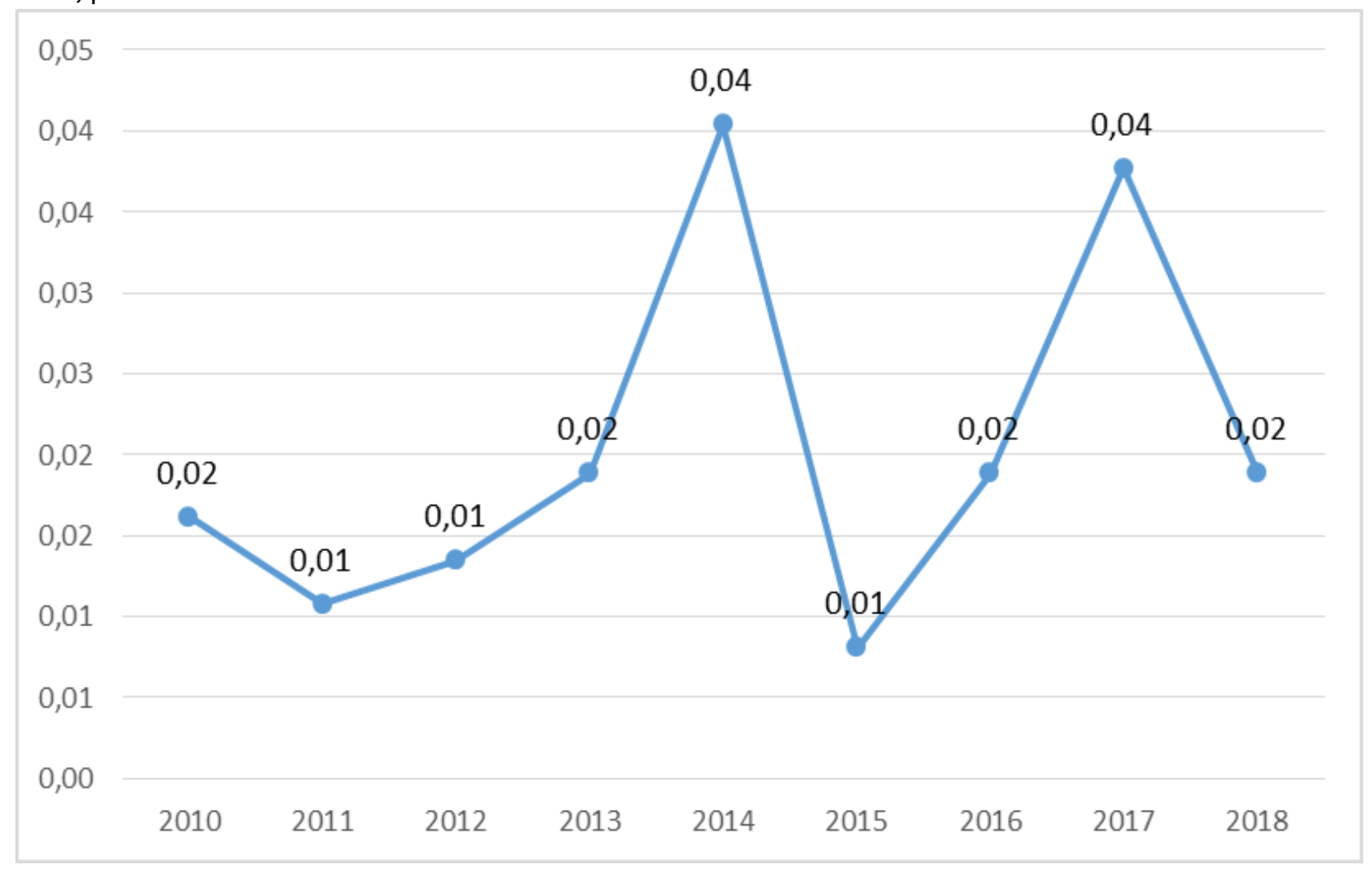

Fonte: MS/DATASUS; Dados consolidados até 30/06/2018

O HIV/Aids é uma realidade nas populações indígenas de Roraima, como também em outros Estados brasileiros. E a maioria dos povos indígenas afirma que essa doença não era do seu mundo e sim os acometeu devido ao contato com as relações de contato com os brancos, ocasionada principalmente por invasores de seus territórios como os grileiros, garimpeiros e os grandes empreendimentos como as barragens hidrelétricas (CARVALHO NS, et al., 2011).

A Tabela 1 demonstra a caracterização da população indígena com AIDS no estado de Roraima no período entre 2010 a 2018. Verifica-se que a maior parte dos casos da epidemia, atinge mais os homens, correspondendo um percentual de $63 \%$ dos casos notificados e $37 \%$ dos casos entre as mulheres.

Percebe-se que a infecção tem acometido mais sexo masculino do que feminino, visto que a faixa etária de maior ocorrência de infecção da doença foi de 20 a 34 anos, ou seja, na população jovem, equivalente a $54 \%$, e 35 a 49 anos com $29 \%$. 
Tabela 1 - Caracterização de casos de AIDS segundo sexo e faixa etária na população indígena do Estado de Roraima no Período de 2010 a 2018.

\begin{tabular}{ccc}
\hline Variável & $\mathbf{N}$ & $\%$ \\
\hline Sexo & & $63 \%$ \\
Masculino & 43 & $37 \%$ \\
Feminino & 25 & \\
\hline Faixa Etária & & $3 \%$ \\
\hline $15-19$ & 2 & $54 \%$ \\
$20-34$ & 37 & $29 \%$ \\
$35-49$ & 20 & $12 \%$ \\
$50-64$ & 8 & $1 \%$ \\
$65-79$ & 1 & \\
Total & 68 &
\end{tabular}

Fonte: MS/DATASUS; Dados consolidados até 30/06/2018.

No Brasil, no ano de 1980 até junho de 2018 houve um percentual de $65,5 \%$ de casos de AIDS em homens e $34,5 \%$ de casos em mulheres. Nos últimos dez anos, observou-se que a taxa de detecção de AIDS em homens apresentou um aumento entre 2007 e 2011 com taxa de 24,8 para 28,3 casos por cem mil habitantes. Quanto à faixa etária, foi observada nos indivíduos com idade entre 25 a 39 anos, em ambos os sexos, que correspondem a $52,6 \%$ de casos do sexo masculino e, entre as mulheres $48,7 \%$ do total dos casos registrados (BRASIL MS, 2018).

No estudo realizado por Kaneta FE e Gomes AM (2011), no período de 2003 a 2006 entre a população indígena do Mato Grosso do Sul observou-se uma predominância maior de Aids entre homens. Outro estudo conduzido entre os povos indígenas no mesmo estado verificou-se que há também uma predominância de casos de AIDS na faixa etária de 20 a 39 anos com 22 (44,0\%) casos, respectivamente (GRAEFF SVB, 2019).

Com isso, constata-se que a maior predominância de Aids está entre o sexo masculino, sendo que as faixas etárias mais acometidas são de 20 a 39 anos, ou seja, a fase adulta. Situação que se repete tanto na população indígena quanto na população não indígena. Diante disso, deve-se haver uma maior atenção com este público alvo para que haja uma redução destes números.

A Tabela 2 demonstra que os maiores percentuais de infecção por HIV/AIDS apresentam-se em indígenas de $1^{\text {a }}$ a $4^{\underline{a}}$ serie incompleta com $25 \%$ de casos, e ensino médio com $16 \%$ de casos, respectivamente. Uma vez que a principal categoria de exposição é a heterossexual, representando $51 \%$ dos casos notificados. Ainda assim, foram notificados casos na categoria homossexual e bissexual.

Tabela 2 - Caracterização de casos de AIDS segundo a escolaridade e categoria de exposição na população indígena do Estado de Roraima no Período de 2010 a 2018.

\begin{tabular}{|c|c|c|}
\hline Variável & $\mathbf{N}$ & $\%$ \\
\hline \multicolumn{3}{|l|}{ Escolaridade } \\
\hline Analfabeto & 7 & $10 \%$ \\
\hline 1a a 4⿳亠丷a série incompleta & 17 & $25 \%$ \\
\hline $4^{a}$ série completa & 3 & $4 \%$ \\
\hline $5^{\mathrm{a}}$ a $8^{\mathrm{a}}$ série incompleta & 9 & $13 \%$ \\
\hline Ensino Fundamental completo & 1 & $1 \%$ \\
\hline Ensino Médio incompleto & 2 & $3 \%$ \\
\hline Ensino Médio completo & 11 & $16 \%$ \\
\hline Ensino Superior incompleto & 6 & $9 \%$ \\
\hline Ensino Superior completo & 2 & $3 \%$ \\
\hline \multicolumn{3}{|l|}{ Categoria de Exposição } \\
\hline Homossexual & 6 & $9 \%$ \\
\hline Bissexual & 6 & $9 \%$ \\
\hline Heterossexual & 51 & $75 \%$ \\
\hline lgnorado & 5 & $7 \%$ \\
\hline
\end{tabular}

Fonte: MS/DATASUS; Dados consolidados até 30/06/2018. 
Sendo assim, pode-se inferir que a escolaridade está ligada à condição social e reflete no acesso à informação e ao conhecimento de meios de prevenção do agravo, assim como de seu tratamento até mesmo para os povos indígenas. Estudos demonstraram que a escolaridade também está diretamente associada à sobrevida de pacientes com HIV/Aids, tendo um efeito protetor contra a maioria das causas de óbito (SILVA JAG, et al., 2015).

Assim, como ocorre em todo país, a principal forma de transmissão do HIV/Aids na população indígena é pela via sexual, sendo que $61 \%$ dos casos notificados estão atribuídos a pessoas que se declaram heterossexuais, $21 \%$ dos casos estão atribuídos a pessoas que se declaram como homo e bissexuais e 6,7\% estão atribuídos a usuários de drogas injetáveis (SANTOS VL, et al., 2010). Diante disso, nota-se que assim como nos demais estados brasileiros, a categoria heterossexual prevalece como grupo vulnerável à exposição do vírus HIV/ AIDS entre a população indígena do Estado de Roraima.

\section{CONSIDERAÇÕES FINAIS}

Verificou-se que a prevalência do HIV/Aids é uma realidade entre os povos indígenas e que devido a sua incidência, e pelo seu estado de cronicidade e por não ter cura o número de casos está aumentando constantemente. Percebe-se que as populações indígenas mais afetadas são as que pertencem aos municípios com o maior contato com a população não indígena. Nota-se também uma subnotificação dos casos de HIV/AIDS em municípios com maior contingente de comunidades indígenas, o que demonstra que a maioria das populações indígenas encontra-se descoberta em relação as sua saúde, o que dificulta para se pensar em planejamento de ações para prevenção, tratamento e promoção da saúde dessa pandemia que ainda é um grande problema de saúde pública. Neste sentido é importante promover ações pertinentes no combate à infecção por HIV/AIDS buscando apoio junto às comunidades indígenas e suas lideranças formas de prevenção assim como também subsidiar um planejamento estratégico da saúde, desenvolvendo excelentes maneiras de educação e promoção da saúde entre esta população.

\section{AGRADECIMENTOS}

Agradeço ao Instituto Insikiran de Formação Superior Indígena por contribuir com a formação dos povos indígenas de Roraima; aos professores do curso Gestão em Saúde Coletiva Indígena em especial as minhas orientadoras Ana Paula e Hosana Carolina por serem pacientes e compreensivas; e a todos que contribuíram direta e indiretamente com o estudo.

\section{REFERÊNCIAS}

1. AMON J, TODRYS K. Acesso de populações migrantes ao tratamento antirretroviral no Sul Global. Sur, Rev. int. direitos humanos. São Paulo. 2009; 6(10): 162-187.

2. ALMEIDA-FILHO N, MEDRONHO RA. Formação Histórica da Epidemiologia. In: MEDRONHO RA, et al. Epidemiologia. - São Paulo: Editora Atheneu, 2009.

3. BASTOS FI. Aids na Terceira Década [online]. Temas em Saúde collection. 20 ed. Rio de Janeiro: Editora Fiocruz, 2006. 104 p. ISBN: 978-85-7541-301-2.

4. BLOCH KV, COUTINHO ESF. Fundamentos da Pesquisa Epidemiológica. In: MEDRONHO RA, et al. Epidemiologia. - São Paulo: Editora Atheneu, 2009.

5. BRASIL MS. Secretaria de Vigilância em Saúde. Boletim Epidemiológico HIV/Aids/2019. Brasília: Ministério da Saúde, Número Especial, Dez. 2019.

6. BRASIL MS. Secretaria de Vigilância em Saúde. Boletim Epidemiológico HIV/Aids de julho de 2017 a junho de 2018. Brasília: Ministério da Saúde, Volume 49. n $53-2018$.

7. BRASIL MS. HIV/Aids, hepatites e outras DST. (Caderno de Atenção Básica, n. 18) (Série A. Normas e Manuais Técnicos). Brasília: Ministério da Saúde, 2006.

8. BRASIL MS. Fundação Nacional de Saúde. Política Nacional de Atenção à Saúde dos Povos Indígenas. $2^{2}$ edição Brasília: Ministério da Saúde. Fundação Nacional de Saúde, 2002. 40. p.

9. BRASIL MS. Secretaria de Políticas de Saúde. Coordenação Nacional de DST e AIDS. Povos indígenas e a prevenção às DST, HIV e AIDS: manual de diretrizes técnicas. Brasília - DF, 2000.

10. CARVALHO F, et al. História, Cultura e Meio Ambiente em Roraima: perspectivas interculturais. Editora da Universidade Federal de Roraima. Boa Vista-RR. 2008; 90 p. 
11. CARVALHO NS, et al. DST em populações indígenas no Brasil: análise crítica e revisão da literatura. DST $\mathrm{j}$ bras doenças sex transm. 2011; 23(3): 142-5.

12. COSTA AJL, KALE PL. Medidas de Frequência de Doença. In: MEDRONHO RA, et al. Epidemiologia. - São Paulo: Editora Atheneu, 2009.

13. GARNELO L, PONTES AL (Org.). Saúde Indígena: uma introdução ao tema. Brasília: MEC-SECADI. 2012; 60-108.

14. GIL AC. Métodos e técnicas de pesquisa social / Antonio Carlos Gil. - 6. ed. - São Paulo: Atlas, 2008.

15. GRAEFF SVB. Aspectos epidemiológicos da infecção pelo HIV e da aids entre povos indígenas Rev Saúde Pública. 2019; 53:71.

16. IBGE. INSTITUTO BRASILEIRO DE GEOGRAFIA E ESTATÍSTICA. CENSO DEMOGRÁFICO 2010. Características da população $\mathrm{e}$ dos domicílios. Resultados do Universo. Disponível em: https://biblioteca.ibge.gov.br/visualizacao/periodicos/93/cd_2010_caracteristicas_populacao_domicilios.pdf Acesso em: 26/06/2019.

17. KANETA FE, GOMES AM. Doenças sexualmente transmissíveis e aids entre indígenas do Distrito Sanitário Especial Indígena do Mato Grosso do Sul de 2001 a 2005 Saúde Coletiva. 2011; 8(47): 7-12.

18. KLEIN CH, BLOCH KV. Estudos Seccionais. In: MEDRONHO RA, et al. Epidemiologia. - São Paulo: Editora Atheneu, 2009.

19. MARINHO MFS. Dos dados a política: a importância da informação em saúde. Epidemiol. Serv. Saúde, Brasília. $2008 ; 17(1): 5-6$.

20. NEGIN J, et al. HIV among Indigenous peoples: a review of literature on HIV-related behaviour since the beginning of epidemic. AIDS Behav. 2015; 19(9): 1720-34.

21. OLIVEIRA EH, et al. Caracterização epidemiológica dos indivíduos portadores do vírus da imunodeficiência humana1 no estado do Piauí, Brasil. 2019; 9(2): e35922002.

22. PEREIRA GFM, et al. HIV/aids, hepatites virais e outras IST no Brasil: tendências epidemiológicas. Rev. bras. epidemiol., São Paulo. 2019; 22(1): e190001.

23. PEREIRA MG. Usos da Epidemiologia. In. PEREIRA MG. Epidemiologia: Teoria e prática. Rio de Janeiro. Guanabara Koogan. 2008.

24. POLEJACK L, SEIDL EMF. Monitoramento e avaliação da adesão ao tratamento antirretroviral para HIV/aids: desafios e possibilidades. Ciência \& Saúde Coletiva. 2010; 15(Supl.1): 1201-1208.

25. POMPEU GVM, SOUSA RJ. O Enfoque das Capacidades e a Responsabilidade Social Empresarial na Promoção do acesso o Trabalho do Migrante No Brasil. RJLB, Ano 5; 2019, 5 643-693.

26. RODRIGUES HAN. Doenças negligenciadas no Estado de Roraima: uma análise a partir da geografia da saúde para o período de 2000 a 2013 - Lajeado, 2015.

27. RORAIMA. SECRETARIA DE ESTADO DA SAÚDE DE RORAIMA. Relatório Anual de Epidemiologia de Roraima 2018. Boa Vista - RR, maio de 2019. Disponível em: https://www.saude.rr.gov.br/cgvs/images/visa/relatorios/relatorioanualdeepidemiologia_2018x.pdf Acesso em: 15 abr. 2020.

28. SANTOS VL, et al. Reflexões sobre as políticas de controle das DST e Aids na população indígena. Revista Tempus Actas em Saúde Coletiva. 2010; 89-100.

29. SILVA JAG, et al. Fatores associados à não adesão aos antirretrovirais em adultos com AIDS nos seis primeiros meses da terapia em Salvador, Bahia, Brasil. Cad. Saúde Pública. 2015; 31(6): 1188-98.

30. SIMÕES GF (Org.). Perfil sócio demográfico e laboral da imigração venezuelana no Brasil. Curitiba: CRV. $2017 ; 112$.

31. SOARES JP, et al. Prevalência e fatores de risco para o HIVIAIDS em populações vulneráveis: uma revisão integrativa de literatura. Arq. Catarin Med. 2017; 46(4): 182-194.

32. SZWARCWALD CL, et al. Estimação da incidência de HIV em dois municípios brasileiros, 2013. Rev. Saúde Pública [online]. 2016; 50.

33. TÁVORA LGF, et al. Hepatitis B, C and HIV co-infections seroprevalence in a Northeast Brazilian center. Arq. Gastroenterol, 2014; 50(4): 277-280. 